\title{
Steps toward interstellar silicate mineralogy
}

\section{Spectral properties and crystallization behaviour of magnesium silicates produced by the sol-gel method ${ }^{\star}$}

\author{
C. Jäger ${ }^{1}$, J. Dorschner ${ }^{1}$, H. Mutschke ${ }^{1}$, Th. Posch ${ }^{2}$, and Th. Henning ${ }^{3}$ \\ 1 Astrophysikalisches Institut und Universitäts-Sternwarte (AIU), Schillergäßchen 2-3, 07745 Jena, Germany \\ 2 Institut für Astronomie, Türkenschanzstraße 17, 1180 Wien, Austria \\ 3 Max-Planck-Institut für Astronomie, Königstuhl 17, 69117 Heidelberg, Germany
}

Received 31 May 2002 / Accepted 4 June 2003

\begin{abstract}
Amorphous silicate particles are generally assumed to be the main dust component in the envelopes of oxygen-rich evolved stars and may be considered the precursors of the pure crystalline enstatite and forsterite particles detected by ISO. We present optical constants in the broad wavelength range $0.2-500 \mu \mathrm{m}$ for a unique series of pure amorphous Mg-silicates $(\mathrm{Mg} / \mathrm{Si}$ in the range $0.7-2.4)$. They have been prepared by the sol-gel process, a chemical technique based on the condensation of $\mathrm{Mg}$ - and Si-hydroxides in a liquid phase. The salient feature of these $\mathrm{Mg}$-silicates is the very small content of $\mathrm{Si}-\mathrm{OH}$ bonds in the silicate network, which considerably reduces the activation energy of crystallization and, thus, decreases the temperature threshold for crystallization as well as crystallization time. The astrophysical relevance of our sol-gel silicates is shown by a comparison of optically thin model spectra based on dust emissivities with ISO-SWS spectra of AGB stars and with $10 \mu \mathrm{m}$ emission profiles of such stars obtained by ground-based spectroscopy. As paradigmatic cases of AGB spectra with respect to the appearance of the silicate bands, TY Dra (slender bands and deep trough between them) and R Cas (broad bands and widely filled-up trough) were used, for which ISO-SWS spectra are available. The dust emissivity derived from TY Dra can be excellently reproduced by the models, suggesting that the dust grains consist indeed of pure amorphous Mg-silicates. Satisfactory agreement was also found with the mean $10 \mu \mathrm{m}$ profiles of some groups of AGB stars and supergiants. Spectra with strong dust emission in the silicate trough like R Cas require additional contributions by other dust components, probably oxides. A rough orientation on the spectral properties of such potential trough opacity contributors has been obtained by subtracting a pure silicate spectrum (TY Dra) from a spectrum with a nearly filled trough and a less pronounced $20 \mu \mathrm{m}$ band (R Cas). In agreement with other amorphous silicates, the spectral index of the new silicate analogues amounts to -2 .
\end{abstract}

Key words. infrared: stars - methods: laboratory - infrared: ISM - stars: circumstellar matter - line: identification

\section{Introduction}

On the basis of ground-based observations and satellite observations (IRAS-LRS spectra), it seemed that cosmic dust silicates were predominantly amorphous. The detailed investigation of the profiles of the 10 and $18-19 \mu \mathrm{m}$ silicate vibrational bands in comparison with laboratory analogs provided a similar result (Dorschner \& Henning 1986, 1995; Dorschner 1999). Additional evidence for the formation of amorphous silicates was provided by non-equilibrium condensation experiments resulting in "chaotic silicates" (Nuth 1996). Consequentially, up to now, amorphous silicates with compositions reflecting the

Send offprint requests to: C. Jäger,

e-mail: conny@astro.uni-jena.de

* Based on observations with ISO, an ESA project with instruments funded by ESA Member States (especially the PI countries: France, Germany, The Netherlands and UK) and with participation of ISAS and NASA. cosmic elemental abundance and the derivation of their optical constants were in the focus of our interest (Jäger et al. 1994; Dorschner et al. 1995; Mutschke et al. 1997). This led to a rather exhaustive characterization of amorphous $\mathrm{Mg}-\mathrm{Fe}-$, $\mathrm{Mg}-\mathrm{Al}-, \mathrm{Ca}-\mathrm{Al}-$, and $\mathrm{Na}-\mathrm{Al}$-silicates, but also to a relative neglect of pure $\mathrm{Mg}$-silicates. The present paper is a contribution to fill this gap.

Spectra obtained by the Infrared Space Observatory (ISO) have surprisingly pointed to clear evidence of crystalline silicates. Observational evidence for the presence of crystalline silicates was found in the spectra of circumstellar dust around Herbig Ae/Be stars (Waelkens et al. 1996), low-mass evolved stars with high mass-loss including planetary nebulae (Waters et al. 1996; Justtanont et al. 1996) and Luminous Blue Variables (LBVs) (Waters et al. 1997). The circumstellar spectra of crystalline silicates exhibited great similarity with the spectrum of comet Hale-Bopp (Crovisier et al. 1997). From the comparison between laboratory spectra of pyroxene- and 
olivine-type silicates and astronomical spectra, it has been concluded that only completely iron-free magnesium silicates like forsterite and enstatite can account for the observed band positions (Jäger et al. 1998; Bowey et al. 2002; Molster et al. 2002b).

Recently, forsterite has been found among the first presolar silicates that were identified in interplanetary dust particles (Messenger et al. 2002) due to a large ${ }^{17} \mathrm{O}$ excess and moderate ${ }^{18} \mathrm{O}$ depletion (which is characteristic of $\mathrm{H}$ burning). This forsterite grain has been proposed to originate from an AGB star. Other silicate grains enriched in ${ }^{17} \mathrm{O}$ and ${ }^{18} \mathrm{O}$ point to an origin in high metallicity stars and type II supernovae. Due to their small sizes, chemically and isotopically similar grains could not be fully characterized, but it has been shown that they are rich in $\mathrm{O}, \mathrm{Si}$ and $\mathrm{Mg}$.

The main dust component in different astronomical environments, though, remains amorphous magnesium-iron silicates (Molster et al. 1999; Demyk et al. 1999; Bouwman et al. 2001; Molster et al. 2002a,b). The crystalline magnesium silicates have probably been formed by thermal annealing. The conversion of amorphous magnesium silicates to crystalline components can be explained by means of annealing processes occurring close to the condensation zones (Hallenbeck et al. 1998; Fabian et al. 2000). However, the annealing temperature necessary to convert the amorphous into the crystalline silicates at a timescale of hours to days is $1000 \mathrm{~K}$. Unless the crystalline $\mathrm{Mg}$-silicates can condense directly from the gas phase, nearly iron-free amorphous silicates should be the precursors of crystalline forsterite and enstatite. Additionally, Rietmeijer et al. (1999) demonstrated the formation of either pure magnesium or iron silicates in vapor-phase condensation experiments. In particular, no mixed $\mathrm{Mg}-\mathrm{Fe}$-silicate grains have been produced in the condensation experiment.

Therefore, we concentrated our experiments on amorphous, iron-free magnesium silicates. This does not mean that we consider Mg-silicates as the only silicate dust component. Mixed Mg-Fe-silicates can be formed after the condensation of $\mathrm{Mg}$-silicates by solid-state reactions with iron or ironcontaining species.

With some exceptions (Dorschner et al. 1995; Scott \& Duley 1996; Hallenbeck et al. 2000), optical constants of amorphous $\mathrm{Mg}$-silicates are rather rare in the literature. It is the aim of this paper to provide optical data of amorphous silicates with varying $\mathrm{MgO} / \mathrm{SiO}_{2}$ ratios which cover the whole wavelength range important for the interpretation of ISO spectra. This investigation includes $\mathrm{Mg}_{0.7} \mathrm{SiO}_{2.7}, \mathrm{MgSiO}_{3}, \mathrm{Mg}_{1.5} \mathrm{SiO}_{3.5}$, $\mathrm{Mg}_{2} \mathrm{SiO}_{4}$, and $\mathrm{Mg}_{2.4} \mathrm{SiO}_{4.4}$. Two of the produced nonstoichiometric materials correspond to the metastable eutectic $\mathrm{Mg}$-silicates compositions $\mathrm{Mg}_{3} \mathrm{Si}_{2} \mathrm{O}_{7}$ and $\mathrm{Mg}_{6} \mathrm{Si}_{8} \mathrm{O}_{22}$ reported to condense preferably in the magnesium silicate binary system by Rietmeijer et al. (1999). Furthermore, the influence of the $\mathrm{Mg} / \mathrm{Si}$ ratio on band positions and strength ratios of the 10 and $20 \mu \mathrm{m}$ bands has been studied. The spectroscopy is supplemented by a careful analytical characterization of the samples.

The sol-gel technique used for the synthesis of magnesium silicate samples is not only interesting for the production of high-melting silicates. It also provides materials bearing chemical properties expected for cosmic silicates. In fact, the formation of magnesium silicates with a small content of isolated $\mathrm{Si}-\mathrm{OH}$ or $\mathrm{Mg}-\mathrm{OH}$ bonds can be considered as highly probable, since $\mathrm{H}_{2} \mathrm{O}$, the most abundant oxygenbearing molecule, participates in the formation of olivine- and pyroxene-type silicates (Gail \& Sedlmayr 1998). These silicate materials show dissenting material properties in comparison to the commonly produced silicates, particularly with regard to their crystallization behaviour. Therefore we completed our experiments with investigations of the annealing behaviour of the synthesized magnesium silicate samples.

\section{Experimental}

The sol-gel process is based on the chemical polymerization of silicates in a liquid phase at low temperatures. Metal organic compounds like tetraethoxysiloxane (TEOS) and magnesium methylate $\mathrm{Mg}\left(\mathrm{OCH}_{3}\right)_{2}$ served as precursors. The metal organic materials have to be soluble in water and alcohol. The polymerization runs in two steps. The first step is the hydrolysis of the precursors and the formation of hydroxides, followed by the condensation of the hydroxides $\left(\mathrm{Si}(\mathrm{OH})_{4}\right.$ and $\left.\mathrm{Mg}(\mathrm{OH})_{2}\right)$. It leads to the formation of a three-dimensional magnesium silicate network. Hydrolysis and condensation are chemical equilibrium reactions which run in competition. In order to avoid phase separations and the formation of inhomogeneous silicate material, it is important to choose silicon and magnesium components with nearly the same hydrolysis and condensation rates.

Compositions up to $0.7 \mathrm{~mol} \mathrm{MgO}$ in the system have been synthesized with magnesium chloride or magnesium nitrate and tetraethoxysiloxane. Metal oxide $(\mathrm{MeO})$ contents larger than $50 \mathrm{Mol} \% \mathrm{MgO}(x \geq 1)$ cannot be produced from anorganic magnesium compounds since they lead to the precipitation of the salt during the reaction (Burlitch et al. 1991).

In order to produce amorphous magnesium silicates $\mathrm{Mg}_{x} \mathrm{SiO}_{2+x}$ (for $x=1 \ldots 2.4$ ), we used an advancement of the $\mathrm{H}_{2} \mathrm{O}_{2}$-assisted sol-gel reaction of magnesium methylate $\left(\mathrm{Mg}\left(\mathrm{OCH}_{3}\right)_{2}\right)$ and TEOS based on the synthesis described by Burlitch et al. (1991) (see also Agladze et al. 1996). The hydrogen peroxide serves as a proton provider and a catalyst. The synthesis has to be performed under a purified Ar-atmosphere to avoid the precipitation of insoluble $\mathrm{Mg}(\mathrm{OH})\left(\mathrm{OCH}_{3}\right)$ and phase separation of $\mathrm{MgO}$. The removal of the solvents methanol and water from the produced gels has been run in a rotary evaporation at reduced pressure. The remaining magnesium silicate powder was heated in order to achieve a densification of the silicate framework and to remove the porosity of the grains. The amorphousness of the samples has been proven by means of X-ray diffraction. The stoichiometry, purity, and phase homogeneity as well as the densification process could be determined by using the Transmission Electron Microscope (TEM) and the Scanning Electron Microscope (SEM) combined with the Electron Dispersive X-ray (EDX) analysis. The samples' compositions were exactly determined by EDX measurements. The compositional results from EDX were carefully compared with corresponding well-analyzed standard samples comprising crystalline and amorphous materials with compositions 
Table 1. Composition of the magnesium silicate samples used for reflection measurements derived by EDX measurements in the SEM and TEM.

\begin{tabular}{cccc}
\hline \hline Sample name & $\mathrm{MgO}$ & $\begin{array}{c}\mathrm{SiO}_{2} \\
\text { wt\% }\end{array}$ & Me-OH \\
\hline $\mathrm{Mg}_{0.7} \mathrm{SiO}_{2.7}$ & 31.12 & 67.74 & 0.87 \\
$\mathrm{Mg}_{1} \mathrm{SiO}_{3}$ & 39.79 & 59.07 & 1.14 \\
$\mathrm{Mg}_{1.5} \mathrm{SiO}_{3.5}$ & 50.31 & 48.71 & 0.98 \\
$\mathrm{Mg}_{2} \mathrm{SiO}_{4}$ & 56.45 & 42.33 & 1.22 \\
$\mathrm{Mg}_{2.4} \mathrm{SiO}_{4.4}$ & 61.34 & 37.89 & 0.77 \\
\hline
\end{tabular}

from $\mathrm{Mg}_{0.5} \mathrm{SiO}_{2.5} \ldots \mathrm{Mg}_{2} \mathrm{SiO}_{4}$. In case of two samples, wetchemical analyses were also performed in order to confirm our EDX results. The homogeneity and purity of the samples were additionally checked by TEM analysis combined with EDX. For an overview of the compositions, see Table 1.

The final powder products have been pressed into very dense pellets at pressures of about $200 \mathrm{t} / \mathrm{cm}^{2}$ load. The pellets were embedded in an epoxide resin and their surfaces were ground and polished. Specular reflectance measurements, at nearly normal incidence, have been performed at the embedded and polished samples from the UV up to the FIR range $(0.2-200 \mu \mathrm{m})$. In the high frequency range a Perkin Elmer Lambda $19 \mathrm{UV} / \mathrm{VIS} / \mathrm{NIR}$ spectrometer, covering the region between 0.2 and $2.5 \mu \mathrm{m}$, was applied. The spectral resolution depends on the wavelength and was $0.5 \mathrm{~nm}$ in the UV/VIS range. An aluminium mirror of calibrated reflectance was used as reference. The IR reflectance measurements in the range between 2 and $300 \mu \mathrm{m}$ were performed by means of a Bruker FTIR spectrometer 113v. In this spectral region a gold mirror reference came into operation. The spectral resolution in the IR was $2 \mathrm{~cm}^{-1}$ which is sufficient for room temperature measurements (Bowey et al. 2001). In the IR and FIR a DTGS/KBr and DTGS/PE detector in combination with $\mathrm{KBr}$ and mylar beamsplitter were used, respectively. The UV/VIS/NIR and IR spectra were merged together without an offset or scaling of the curves. A part of the powder samples has been used for transmission measurements of $\mathrm{KBr}$ and $\mathrm{PE}$ pellets in the IR range ( 2 to $300 \mu \mathrm{m}$ ). For this purpose, grains smaller than $1 \mu \mathrm{m}$ were produced by additional grinding and sedimentation of the grains in water-free alcohol.

\section{Results}

\subsection{Spectral properties}

The results of the transmission measurements of the magnesium silicate sol-gel sample are shown in Fig. 1. In the binary system $\mathrm{MgO} \cdot \mathrm{SiO}_{2}$, the effect of the $\mathrm{MgO} / \mathrm{SiO}_{2}$ ratios on the position and shape of the $\mathrm{Si}-\mathrm{O}$ vibrational bands is demonstrated. Amorphous magnesium silicates typically show two broad bands at about 10 and $20 \mu \mathrm{m}$ corresponding to $\mathrm{Si}-\mathrm{O}$ stretching and bending vibrations. The large width of both bands results from a distribution of bond lengths and angles within the amorphous structure. The $20 \mu \mathrm{m}$ band is additionally broadened due to the coupling of the $\mathrm{Si}-\mathrm{O}$ bending to the $\mathrm{Mg}-\mathrm{O}$ stretching vibration in this spectral region. The coupling has been reported

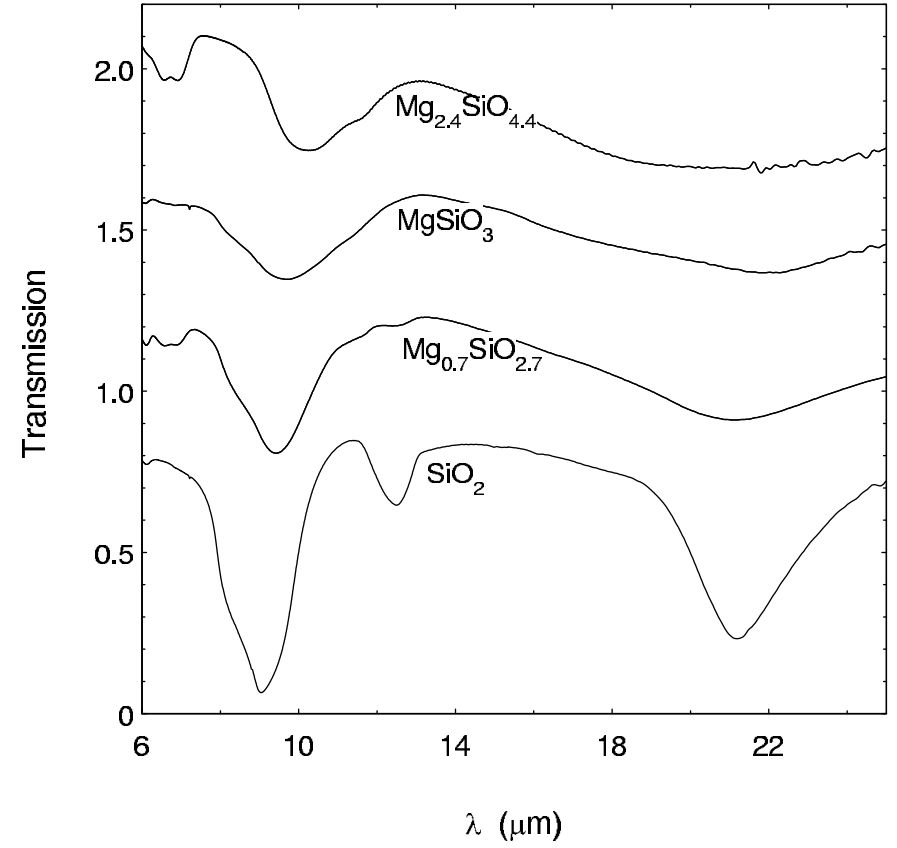

Fig. 1. IR transmission spectra of magnesium silicates in the binary system $\mathrm{MgO} \cdot \mathrm{SiO}_{2}$ for various $\mathrm{MgO} / \mathrm{SiO}_{2}$ ratios. The average particle size is less than $1 \mu \mathrm{m}$. For the sake of clarity, the curves have been successively shifted by 0.4 .

e.g. by Gervais et al. (1987) leading to a combined band of a huge width. The incorporation of $\mathrm{Mg}$ into the silicate network can occur in two different ways, as network former (4-fold coordination) and/or as network modifier (6-fold coordination).

The appearance of a third band at $12.5 \mu \mathrm{m}$, visible in the $\mathrm{SiO}_{2}$ spectrum, is typical for $\mathrm{SiO}_{2}$ and disappears at a $\mathrm{MgO}$ content of 0.5 . The band can be assigned to the symmetric stretching vibration of $\mathrm{Si}-\mathrm{O}-\mathrm{Si}$ bonds or to ring structures (Fuxi 1979; Brinker \& Scherer 1990). The small double band at about $7 \mu \mathrm{m}$ is caused by traces of magnesium carbonate which can be formed during grinding. In order to separate large grains $(\leq 1 \mu \mathrm{m})$ from the powder sample we used the sedimentation technique which lead to a slight enrichment of the carbonate in the sample because of the smaller specific weight of the carbonate grains. By comparison of the integral intensity of the 7 micron band with selected carbonate samples we could roughly estimate the carbonate content to $6 \mathrm{~mol} \%$. The carbonate impurity was only found in the ground and sedimented magnesium silicate sample which was used for the transmission measurement and is not an impurity in the reflection samples.

The position of the $\mathrm{Si}-\mathrm{O}$ stretching vibration is shifted from $9 \mu \mathrm{m}$ for the pure $\mathrm{SiO}_{2}$ to $9.7 \mu \mathrm{m}$ for $\mathrm{MgSiO}_{3}$ and $10.25 \mu \mathrm{m}$ for the $\mathrm{Mg}_{2.4} \mathrm{SiO}_{4.4}$. The influence of the $\mathrm{MgO}$ content on the position of the $\mathrm{Si}-\mathrm{O}$ bending vibration groups is difficult to observe since the widths of the $20 \mu \mathrm{m}$ bands rise strongly with increasing $\mathrm{MgO}$ content. The position of this feature seems to remain constant. This effect is related to morphological and matrix effects in the $\mathrm{KBr}$. The $\mathrm{KBr}$ environment leads to a broadening and a long-wavelength shift of both peaks. However, the matrix influence is much stronger for the very broad $20 \mu \mathrm{m}$ bands. We assume that different bonds within the silicate network such as $\mathrm{Si}-\mathrm{O}$ and $\mathrm{Mg}-\mathrm{O}$ will be differently influenced by $\mathrm{KBr}$. 


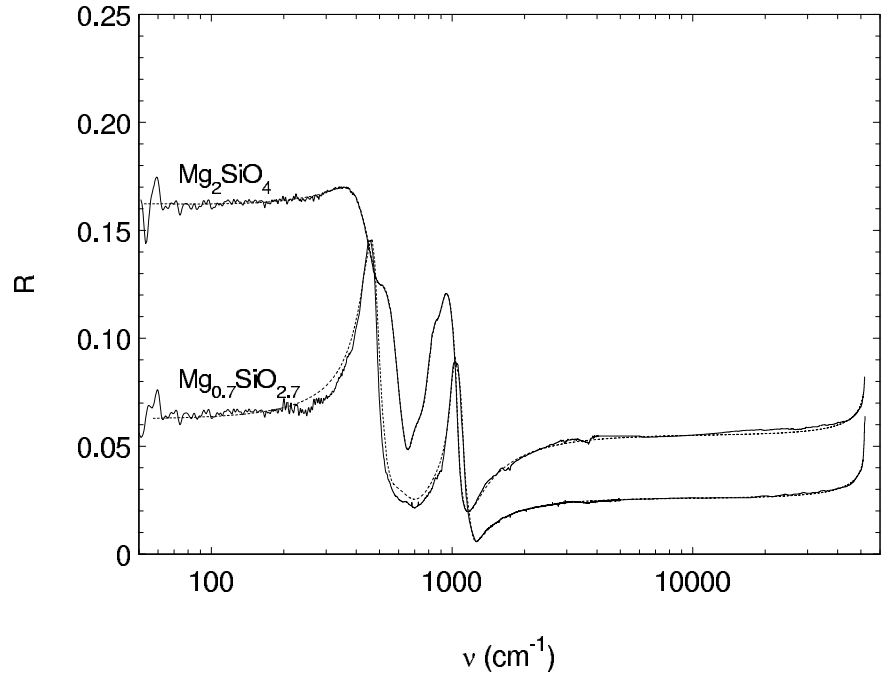

Fig. 2. Comparison between the calculated reflection spectra (solid lines) and the measured reflection spectra (dashed lines) demonstrated for two silicates.

\subsection{Reflectance spectra}

Two typical reflectance spectra of magnesium silicates are shown in Fig. 2. There are two pronounced silicate vibrational bands at 1000 and $500 \mathrm{~cm}^{-1}$ in all spectra discernible. In the FIR the spectra behave differently. $\mathrm{Mg}_{0.7} \mathrm{SiO}_{2.7}$ shows a continuous decline of reflectivity with decreasing wavenumber starting at about $460 \mathrm{~cm}^{-1}$. In the spectra of the samples $\mathrm{MgSiO}_{3}$ up to $\mathrm{Mg}_{2.4} \mathrm{SiO}_{4.4}$ a nearly constant reflectivity can be observed in the FIR, which can be seen in the reflectance spectrum of $\mathrm{Mg}_{2.4} \mathrm{SiO}_{4.4}$ as a representative of this group. This FIR behavior will be discussed in the next section. The rising $\mathrm{MgO}$ content in the silicates also results in a shift of the 10 and $20 \mu \mathrm{m}$ bands and an increase of band widths. $\mathrm{Mg}_{2} \mathrm{SiO}_{4}$ displays a clear long-wavelength shift of the $\mathrm{Si}-\mathrm{O}$ stretching band and the broadening of the $\mathrm{Si}-\mathrm{O}$ bending vibration compared to $\mathrm{Mg}_{0.7} \mathrm{SiO}_{2.7}$. The general reflectivity from the $\mathrm{UV}$ up to the FIR is as well growing with increasing $\mathrm{MgO} / \mathrm{SiO}_{2}$ ratios in the samples. This is especially evident in the VIS and NIR ranges where the reflectivity is nearly constant. The UV reflectance is stamped by a strong rise of the reflectivity beyond $200 \mathrm{~nm}\left(50000 \mathrm{~cm}^{-1}\right)$ due to electronic interband transitions. This behavior is typical for pure magnesium silicates since no absorbing elements (transition elements) with electronic transitions in the VIS and NIR region are contained. Very small structures in the reflectance spectra at about $3660 \mathrm{~cm}^{-1}$ are caused by $\mathrm{O}-\mathrm{H}$ stretching vibrations of isolated $\mathrm{Si}-\mathrm{OH}$ or $\mathrm{Mg}-\mathrm{OH}$ groups.

\subsection{Derivation and characteristics of the optical constants}

Optical constants $(n, k)$ of the produced stoichiometric and nonstoichiometric magnesium silicates have been derived from the reflection measurements (see Fig. 3). For $\mathrm{Mg}_{0.7} \mathrm{SiO}_{2.7}$ the Lorentz-oscillator fit method was exclusively used to determine the complex refractive index in the whole measurement range from the UV up to the FIR. For the other magnesium silicates $n$ and $k$ were evaluated by a combination of Kramers-Kronig analysis (KKR) and Lorentz-oscillator fit method. Concretely, we performed a KKR analysis in the IR range from 2 to $200 \mu \mathrm{m}$. The FIR tail starting from $200 \mu \mathrm{m}$ and the NIR tail starting from $2 \mu \mathrm{m}$ were extrapolated assuming that the reflectivities converge to constant values at zero and infinite wavelengths, respectively. The fulfillment of the causality criterion

$\int_{-\infty}^{\infty} \ln (R(\omega) / R(\infty)) \mathrm{d} \omega=0$

was verified. Note that most of the resulting $n(\omega)$ spectra (see Fig. 3) are characterized by a monotonous decrease between $\omega=0$ and the shortest resonance frequency, or, in other words, by a monotonous increase of $n(\lambda)$ between the largest resonance wavelength and $\lambda \rightarrow \infty$. It can be analytically shown that for strongly damped oscillators - i.e. for a damping constant $\Gamma_{\text {le }}$ of the lowest energy oscillator which fulfills the condition $\Gamma_{\mathrm{le}}>\omega_{\mathrm{le}}$ (where $\omega_{\mathrm{le}}$ is the lowest energy oscillator frequency) $-n$ must indeed monotonously decrease between $\omega=20$ and $\omega=\omega_{\mathrm{le}}$. For one of our amorphous Mg silicates, namely $\mathrm{Mg}_{0.7} \mathrm{SiO}_{2.7}$, it turned out that $\Gamma_{\mathrm{le}}<\omega_{\mathrm{le}}$ ); therefore, its n curve resembles the "canonical" shape of a narrow Lorentz oscillator profile. By contrast, all the other samples represent the case of the very strongly damped oscillator system with $\Gamma_{\mathrm{le}}>\omega_{\mathrm{le}}$. The observed strong broadening of the 20 micron bands of amorphous magnesium silicates is on one hand caused by the disorder in an amorphous solid leading to a distribution of bond angles and distances and therefore to a superposition of many slightly different vibrational frequencies but on the other hand by the coupling of the $\mathrm{Si}-\mathrm{O}$ bending to the $\mathrm{Mg}-\mathrm{O}$ stretching vibration in this spectral region. The coupling, reported by Gervais et al. (1987), give rise to the formation of a combined band with a huge width ranging from about 200 to $700 \mathrm{~cm}^{-1}$.

In the astronomical literature, the properties of these very broad oscillator profiles have been rarely addressed hitherto. However, the optical constants derived for different amorphous cosmic dust analogs are generally characterized by the condition $\Gamma_{\mathrm{le}}>\omega_{\mathrm{le}}$ and the corresponding broad profile (see e.g. Eriksson et al. 1981; Draine \& Lee 1984; Ossenkopf et al. 1992; Dorschner et al. 1995; Begemann et al. 1997).

KKR analysis could not be used in the UV/VIS/NIR region, since at the short wavelength side the spectra end in the region of electronic transitions, whereas the KKR method requires a negligible absorption at both spectral limits. Therefore, the Lorentz-oscillator fit method has been applied to calculate the $n$ and $k$ values below $14 \mu \mathrm{m}$. As for the applicability of this method, we refer to the monographs by Wooten (1972) and Bohren \& Huffman (1983), who show that in the UV/VIS range, Lorentz oscillator fits can be used to model all direct interband transitions in insulators. In order to model the tail of the electronic transitions in our magnesium silicates we used 2 oscillators, a broad and very strong one at about $60000 \mathrm{~cm}^{-1}$ representing the expected strong electronic transitions below $190 \mathrm{~nm}$ and being mainly responsible for the refractive index in the visible. A second oscillator at about $53000 \mathrm{~cm}^{-1}$ was used to model the fine structure of the absorption edge in the 
Table 2. Diagnostic spectral parameters of the 10 and $20 \mu \mathrm{m}$ bands of amorphous magnesium silicates based on calculations of the absorption cross section per unit volume $C /\langle V\rangle$ (in $\mathrm{cm}^{-1}$ ) for small spheres (Sph.) and a continuous distribution of ellipsoids (CDE) for particles in the Rayleigh limit. $\lambda$ and $A$ represent the peak position in $\mu \mathrm{m}$ and the absorption cross section per unit volume $C /\langle V\rangle$ in $\mathrm{cm}^{-1}$, respectively. $W$ stands for the FWHM of the bands. The band ratio has been calculated by dividing the intensity of the $20 \mu \mathrm{m}$ band by the intensity of the $10 \mu \mathrm{m}$ band.

\begin{tabular}{|c|c|c|c|c|c|c|c|c|c|c|c|c|c|c|}
\hline \multirow{3}{*}{ Comp. } & \multicolumn{6}{|c|}{$\bar{~} 10 \mu \mathrm{m}$} & \multicolumn{6}{|c|}{$20 \mu \mathrm{m}$} & \multirow{2}{*}{\multicolumn{2}{|c|}{$\begin{array}{c}\text { band ratio } \\
A_{20} / A_{10}\end{array}$}} \\
\hline & \multicolumn{2}{|c|}{$\lambda_{10}[\mu \mathrm{m}]$} & \multicolumn{2}{|c|}{$A_{10}\left[\mathrm{~cm}^{-1}\right]$} & \multicolumn{2}{|c|}{$W_{10}[\mu \mathrm{m}]$} & \multicolumn{2}{|c|}{$\lambda_{20}[\mu \mathrm{m}]$} & \multicolumn{2}{|c|}{$A_{20}\left[\mathrm{~cm}^{-1}\right]$} & \multicolumn{2}{|c|}{$W_{20}[\mu \mathrm{m}]$} & & \\
\hline & Sph. & $\mathrm{CDE}$ & Sph. & $\mathrm{CDE}$ & Sph. & $\mathrm{CDE}$ & Sph. & $\mathrm{CDE}$ & Sph. & $\mathrm{CDE}$ & Sph. & $\mathrm{CDE}$ & Sph. & $\mathrm{CDE}$ \\
\hline $\mathrm{Mg}_{0.7} \mathrm{SiO}_{2.7}$ & 9.15 & 9.20 & 6945 & 7043 & 1.41 & 1.56 & 20.21 & 20.86 & 3413 & 3825 & 5.61 & 5.68 & 0.49 & 0.54 \\
\hline $\mathrm{MgSiO}_{3}$ & 9.26 & 9.37 & 6165 & 6642 & 1.88 & 2.10 & 19.41 & 20.22 & 2520 & 3023 & 8.53 & 10.0 & 0.41 & 0.46 \\
\hline $\mathrm{Mg}_{1.5} \mathrm{SiO}_{3.5}$ & 9.62 & 9.74 & 6725 & 6992 & 1.99 & 2.41 & 18.11 & 18.70 & 3533 & 4020 & 9.91 & 13.3 & 0.52 & 0.57 \\
\hline $\mathrm{Mg}_{2} \mathrm{SiO}_{4}$ & 9.80 & 9.91 & 6923 & 7395 & 2.05 & 2.43 & 17.42 & 17.90 & 3165 & 3698 & 10.0 & 13.0 & 0.47 & 0.50 \\
\hline $\mathrm{Mg}_{2.4} \mathrm{SiO}_{4.4}$ & 10.07 & 10.2 & 4778 & 5198 & 2.66 & 2.80 & 17.11 & 17.76 & 3925 & 4395 & 9.83 & 13.1 & 0.82 & 0.84 \\
\hline
\end{tabular}

range between 210 and $190 \mathrm{~nm}$ for each individual composition of magnesium silicates. The $10 \mu \mathrm{m}$ IR band has been included in the modeling using 2-4 oscillators in order to achieve an overlap of the results obtained by KKR and Lorentz-oscillator fitting. It turned out that Lorentz-oscillator model was better suited to represent the tail of the infrared bands below $8 \mu \mathrm{m}$. Therefore, we merged both results at this spectral position.

The validity of our calculations is demonstrated by the comparison between the original reflectance spectra of the silicates and the calculated reflection determined from the optical constants shown in Fig. 3. The measured and calculated reflection curves, displayed in Fig. 2, show a very good coincidence. Only small deviations in the band region of the $\mathrm{Mg}_{0.7} \mathrm{SiO}_{2.7}$ spectrum can be seen, which result from the difficulty to reproduce the broad vibrational bands of amorphous samples with Lorentz-oscillators. Efimov (1999) has compared quantitative analysis methods of IR spectra of various inorganic glasses to determine optical constants and found that the Kramers-Kronig transformation and the Lorentzoscillator fit method provide practically coinciding optical constants. KKR can have advantages compared to the oscillator-fitmethod since KKR involves no microphysical model for the response of solid material to the electromagnetic field. That means that KKR is not correlated to any particular band shape, which guarantees the practicability of this relation to spectra independent of the frequency range and the materials' properties. Therefore, KKR is a generally accepted method for the determination of optical constants of solids including amorphous materials (Gaskell \& Johnson 1976; Kamitsos et al. 1995; Hudgens \& Martin 1996; Aasland et al. 1997).

The knowledge of the optical constants enables us to calculate the absorption cross section per unit volume of grains with different morphology (see Table 2 and Fig. 12). The calculated absorption cross sections have shown that $\mathrm{MgO}$ influences the position of the 10 and $20 \mu \mathrm{m}$ band. With increasing $\mathrm{MgO}$ content, the $10 \mu \mathrm{m}$ band is shifted to longer wavelengths, whereas the $20 \mu \mathrm{m}$ band is shifted in the opposite direction. This result is in agreement with the findings of McMillan (1984), who demonstrated the influence of alkaline and alkaline earth oxides on the positions of the stretching and bending $\mathrm{Si}-\mathrm{O}$ modes in silicate glasses and melts. The positions of the 10 and $20 \mu \mathrm{m}$ bands are sensitive to the number of bridging oxygen atoms and therefore to the degree of polymerization of $\mathrm{SiO}_{4}$ tetrahedra in the amorphous silicate network. The $10 \mu \mathrm{m}$ bands

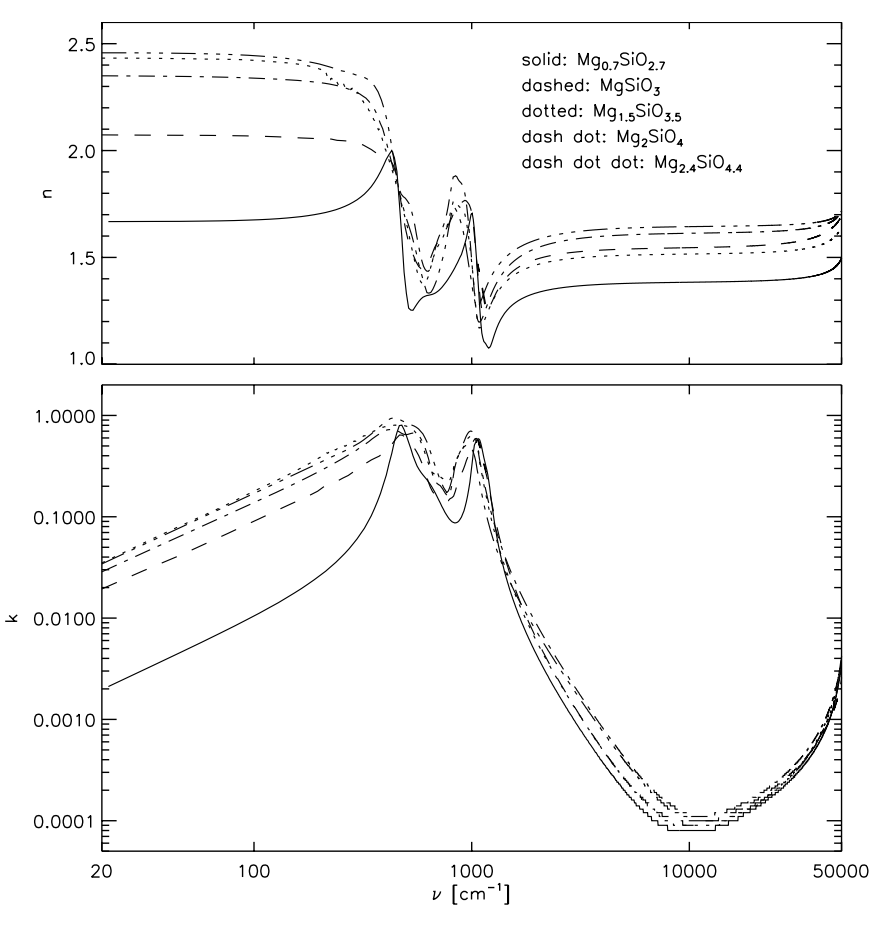

Fig. 3. Optical constants of amorphous magnesium silicates of varying composition derived from reflection measurements by KKR and Lorentz oscillator fit methods. The $n$ - and $k$-data files can be taken from the internet homepage of the Astrophysical Institute and University Observatory Jena (http://www.astro.uni-jena.de/ Laboratory/Database/silicates.html).

are broadened due to the superposition of individual bands of $\mathrm{Si}-\mathrm{O}$ bonds, whereas the huge increase of the $20 \mu \mathrm{m}$ band widths' are caused by superposition of $\mathrm{Si}-\mathrm{O}$ and $\mathrm{Mg}-\mathrm{O}$ bonds, varying in bond lengths and angles. Differences to measured transmission spectra of small grains in $\mathrm{KBr}$, especially in the range of the $20 \mu \mathrm{m}$ band, result from matrix and morphological effects in the $\mathrm{KBr}$ pellets.

\subsection{Comparison with other amorphous magnesium silicates}

Figure 4 shows the comparison of our $n$ and $k$ data with optical data of pyroxene and olivine glasses produced by melting and quenching techniques (Dorschner et al. 1995). The spectral behaviour in the UV/VIS range is influenced by the 


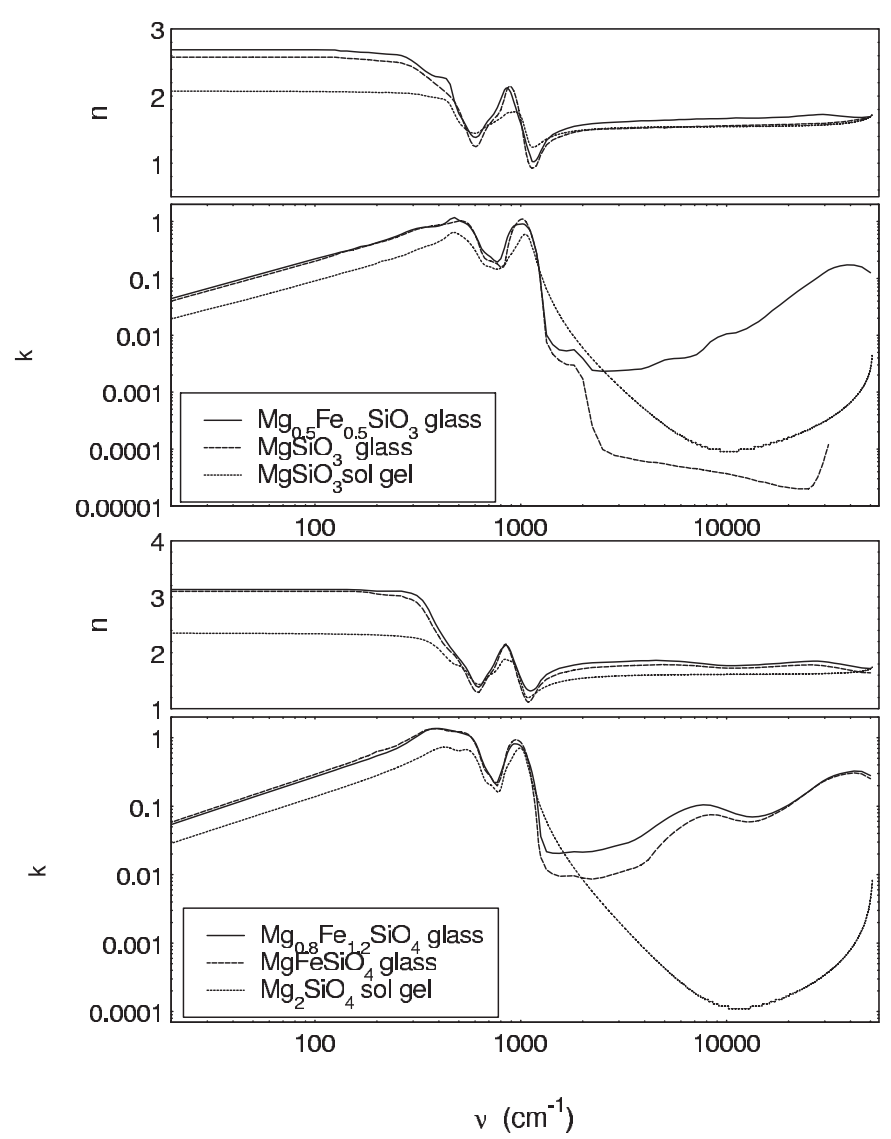

Fig. 4. Comparison of the optical data of $\mathrm{Mg}_{2} \mathrm{SiO}_{4}$ and $\mathrm{MgSiO}_{3}$ produced by the sol-gel method with those of iron-containing proxeneand olivine glasses.

presence of transition metal ions. The incorporation of iron in glasses leads to a series of electronic transitions from the UV up to the NIR range which is well discernible in the ironcontaining glasses in Fig. 4. It is well known that in pure magnesium silicate glasses the UV absorption edge is determined by the interaction of UV radiation with electrons of bridging and non-bridging oxygen. The strong and rather broad absorptions at wavelengths smaller than $200 \mathrm{~nm}$ are caused by electronic transitions, concretely, by excitons and interband transitions between the $2 \mathrm{p}$ orbitals of oxygen and the $3 \mathrm{~d}$ orbitals of silicon (Liepmann \& Neuroth 1995; Fuxi 1979). Since the nonbridging oxygen are less tightly bound to silicon than the bridging oxygens their electronic transitions occur at longer wavelengths than those of bridging oxygen.

The UV absorption edge of the sol-gel product $\mathrm{MgSiO}_{3}$ is shifted to shorter wavelengths $(200 \mathrm{~nm})$ compared to the iron-free $\mathrm{MgSiO}_{3}$ glass. The short-wavelength shift of the $\mathrm{UV}$ absorption edge is probably related to the incorporation of $\mathrm{Mg}^{2+}$ ions into the silicate as a network former avoiding the formation of non-bridging oxygen. This is also confirmed by the fact that the $\mathrm{MgO}$ content in the sol-gel silicates does not have any influence on the absorption edge. Small differences between the samples can be raised by varying $\mathrm{Si}-\mathrm{OH}$ bonds. The $n$ and $k$ values of the sol-gel silicates are distinctly smaller than those of magnesium silicate glasses, resulting in differences of the absolute values of the absorption coefficients for
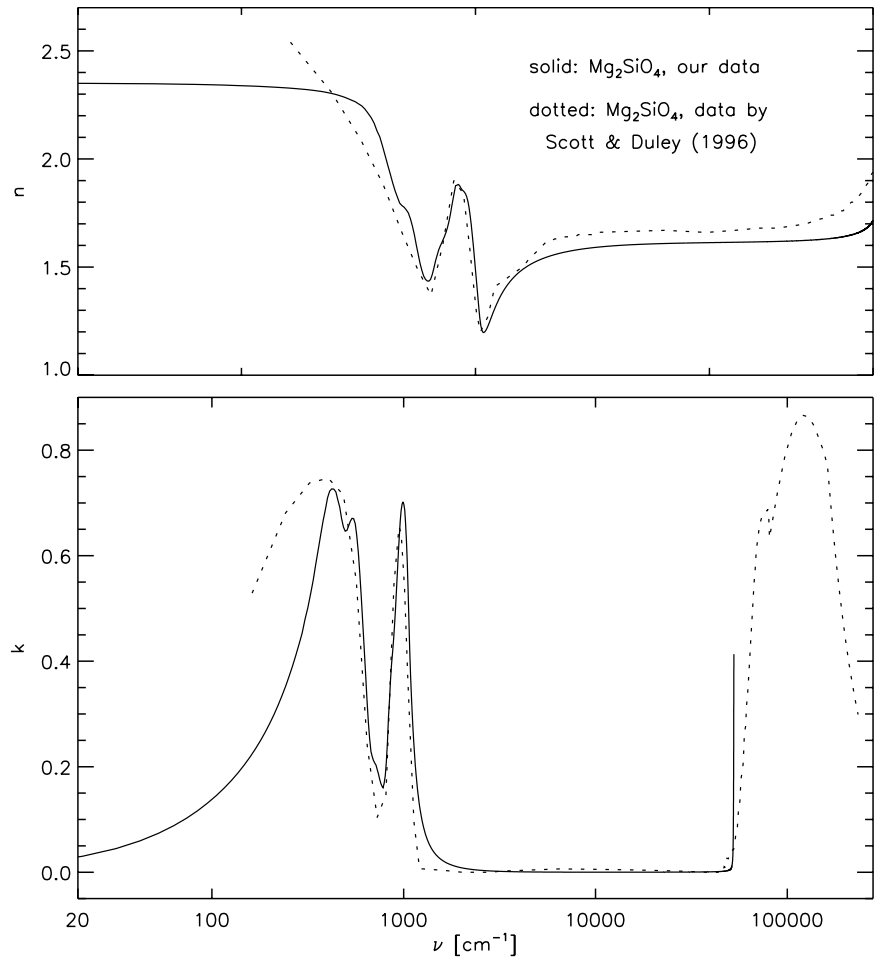

Fig. 5. Comparison of the optical constants of amorphous $\mathrm{Mg}_{2} \mathrm{SiO}_{4}$ produced by sol-gel method (solid line) and optical data of $\mathrm{Mg}_{2} \mathrm{SiO}_{4}$ films (dotted line) derived by Scott \& Duley (1996).

spheres and CDE. The position of the $10 \mu \mathrm{m}$ band is shifted to shorter wavelengths by $0.06 \mu \mathrm{m}$. The calculated bending mode position for $\mathrm{MgSiO}_{3}$ spheres in the Rayleigh limit is shifted to longer wavelengths $(19.4 \mu \mathrm{m})$ compared to the calculated position for $\mathrm{MgSiO}_{3}$ glass spheres $(17.7 \mu \mathrm{m}$; Dorschner et al. 1995).

However, the comparison with optical data of a $\mathrm{Mg}_{2} \mathrm{SiO}_{4}$ film (Scott \& Duley 1996) produced by gas phase condensation shows a good agreement with the optical data of $\mathrm{Mg}_{2} \mathrm{SiO}_{4}$ produced by the sol-gel technique (see Fig. 5). There is a satisfactory agreement in the run of the $n$ and $k$ curves especially in the UV/VIS range. Slight differences, according to structural variety in the amorphous structure of the differently produced magnesium silicates, are discernible in the MIR range. For spherical particles the data by Scott \& Duley (1996) yield maxima of the absorption cross section at 10.1 and $18.6 \mu \mathrm{m}$ compared to our values of 9.8 and $17.4 \mu \mathrm{m}$ (see Fig. 6). The comparison between the differently produced magnesium silicates demonstrates the existence of varying amorphous material with differences in the internal structures. Based upon these results, one has to conclude that the amorphous state of any magnesium silicate is not unique but there exist different possibilities for structural arrangements of subunits in the amorphous silicate structure, similar to the different structures of amorphous carbon with strongly varying optical properties.

One can draw the conclusion that just as in the case of amorphous carbon, different structures with varying optical constants may exist and that the short-range as well as 
Table 3. Activation energies of crystallization $E_{\mathrm{a}} / k$ determined for differently produced magnesium silicates.

\begin{tabular}{cccc}
\hline \hline Material & Material properties & $E_{\mathrm{a}} / k(\mathrm{~K})$ & Crystalline phase \\
\hline $\mathrm{Mg}_{2} \mathrm{SiO}_{4}$ & Smoke, inhomogeneous & 39100 & forsterite \\
$\mathrm{MgSiO}_{3}$ & Smoke, inhomogeneous & 42040 & forsterite, tridymite \\
$\mathrm{MgSiO}_{3}$ & Glas, homogeneous, grain sizes $\leq 1 \mu \mathrm{m}$ & $40100-43300$ & \\
$\mathrm{Mg}_{0.95} \mathrm{Fe}_{0.05} \mathrm{SiO}_{3}$ & glass, homogeneous, grain sizes $\leq 1 \mu \mathrm{m}$ & 38525 & enstatite \\
& & & \\
$\mathrm{Mg}_{-} \mathrm{silicate}$ & Sol-gel, homogeneous, $\mathrm{Me}-\mathrm{OH}$ bondings & $26600-31700$ & \\
$\mathrm{MgSiO}_{3}$ & & & enstatite \\
$\mathrm{Mg}_{1.5} \mathrm{SiO}_{3.5}$ & & dependent on & enstatite, forsterite \\
$\mathrm{Mg}_{1.8} \mathrm{SiO}_{3.8}$ & forsterite \\
$\mathrm{Mg}_{2} \mathrm{SiO}_{4}$ & & & forsterite \\
$\mathrm{Mg}_{2.4} \mathrm{SiO}_{4.4}$ & & & forsterite \\
$\mathrm{Mg}_{2.6} \mathrm{SiO}_{4.6}$ & & & forsterite, $\mathrm{MgO}$ \\
\hline
\end{tabular}

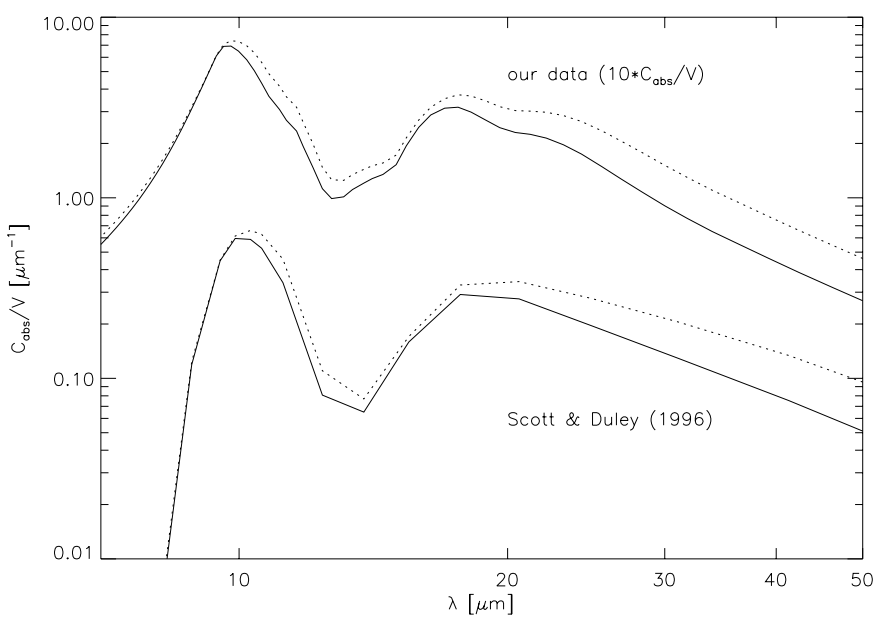

Fig. 6. Comparison of the absorption cross section of amorphous $\mathrm{Mg}_{2} \mathrm{SiO}_{4}$ produced by sol-gel method with that of $\mathrm{Mg}_{2} \mathrm{SiO}_{4}$ films calculated from the $n$ and $k$ data by Scott \& Duley (1996). The solid lines stand for spherical particles, whereas the dotted lines represent the results for a continuous distribution of ellipsoids. The cross sections derived from our data have been offset by a factor of 10 .

the medium-range order determine their optical properties in the UV/VIS and in the IR region.

\subsection{Crystallization behaviour and the role of $\mathrm{Si}-\mathrm{OH}$ groups}

It is generally known that a phase transition between crystalline and amorphous silicates leads to a dramatic change of their spectral properties from the UV/VIS to the FIR region. Phase transitions can be caused by annealing at sufficiently high temperatures. Crystallization is a complex process including nucleation and crystal growth.

In this paper we use an empirical approach for the description of the crystallization enclosing both processes. Infrared spectroscopy has been used for monitoring the progress of annealing. The characteristic annealing time $\tau$ is determined by the appearance of first hints of fine structure indicating the increase of structural order. The activation energy of crystallization for the pure sol-gel produced magnesium silicates has been calculated using the equation $\tau^{-1}=v_{0} \mathrm{e}^{-E_{\mathrm{a}} / k T}$, wherein $E_{\mathrm{a}}$ is the activation energy and $v_{0}$ a constant proportional to the mean vibrational frequency of the silicate lattice $\left(v_{0}=2.0 \times 10^{13} \mathrm{~s}^{-1}\right.$; Gail \& Sedlmayr 1998; Lenzuni et al. 1995).

The results of the annealing experiments are summarized in Table 3. For the sake of comparison, the table additionally contains activation energies of differently produced silicate materials like glasses and smokes (Fabian et al. 2000). The activation energies $E_{\mathrm{a}} / k$ of magnesium silicates produced by sol-gel synthesis amount to values between 26600 and $31700 \mathrm{~K}$ corresponding to about two thirds of the crystallization activation energy of $\mathrm{Mg}$-silicate glasses and smokes. Corresponding annealing temperatures were between 723 and $873 \mathrm{~K}$. Annealing experiments with sol-gel produced magnesium silicates demonstrate that $E_{\mathrm{a}}$ is independent of the composition of the materials. This unusual behaviour has its seeds in a content of non-associated $\mathrm{Si}-\mathrm{OH}$ and $\mathrm{Mg}-\mathrm{OH}$ bonds stemming from the sol-gel synthesis.

Non-associated $\mathrm{Si}-\mathrm{OH}$ and $\mathrm{Mg}-\mathrm{OH}$ bonds give rise to a narrow band at about $2.7 \mu \mathrm{m}$ due to $\mathrm{O}-\mathrm{H}$ stretching vibrations (see Fig. 7). These groups can remain from the condensation process. However, the band position of $3670 \mathrm{~cm}^{-1}$ rather points to an excess of $\mathrm{Si}-\mathrm{OH}$ bonds (Iler 1979a; Morimoto et al. 1999) compared to $\mathrm{Mg}-\mathrm{OH}$ since the latter have a band at $3700 \mathrm{~cm}^{-1}$ (Mitra 1962; Shinoda et al. 2002). In case of reduced temperature crystallization this narrow band at $2.7 \mu \mathrm{m}$ has been found in addition to the first spectroscopic hints to crystallization due to a content of non-associated $\mathrm{O}-\mathrm{H}$ bonds in these samples. The content of these $\mathrm{O}-\mathrm{H}$ bonds has been spectroscopically determined by comparison of the $2.7 \mu \mathrm{m}$ band with a sample of well known $\mathrm{Si}-\mathrm{OH}$ content. The latter does not exceed $1.3 \mathrm{wt} \%$ that remained from the sol-gel synthesis. Isolated $\mathrm{O}-\mathrm{H}$ bonds in a magnesium silicate framework reduce the formation of oxygen bridges between the $\mathrm{SiO}_{4}$ tetrahedra and form non-bridging oxygen. The increase of non-bridging oxygen leads to a decrease of the viscosity (Scholze 1988). The influence of $\mathrm{OH}$ groups is comparable to that of alkaline ions which act as network modifier introducing non-bridging 


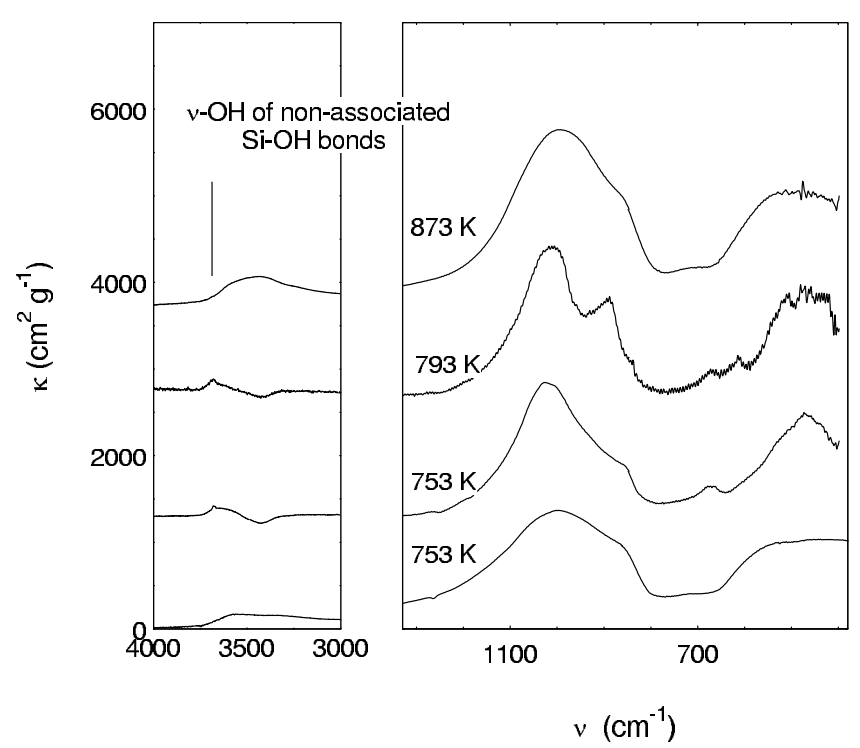

Fig. 7. Powder transmission spectra of $\mathrm{Mg}_{2} \mathrm{SiO}_{4}$ (in $\mathrm{KBr}$ pellets) with varying $\mathrm{Si}-\mathrm{OH}$ content. All the samples have been annealed for $1 \mathrm{~h}$. The spectra clearly show the correlation between crystallization and the appearance of the characteristic vibrational band of isolated $\mathrm{Si}-\mathrm{OH}$ bonds located at $2.7 \mu \mathrm{m}$. The spectra have been vertically offset.

oxygen (Iler 1979b). This is easy to understand since the proton $\mathrm{H}^{+}$of the $\mathrm{OH}$ group can be considered like a alkaline ion. Thereby the viscosity of the magnesium silicates is decreased and the crystallization temperature can be lowered by 200 to $300 \mathrm{~K}$ (cf. Fig. 7). That means that the crystallization temperature is dependent on the $\mathrm{Si}-\mathrm{OH}$ content. A further incorporation of $\mathrm{Si}-\mathrm{OH}$ does not lead to a further decrease of the crystallization temperature. The temperature of crystallization can be decreased from $1000 \mathrm{~K}$ for $\mathrm{Si}-\mathrm{OH}$ free material to $730 \mathrm{~K}$ for magnesium silicate containing $1.3 \mathrm{wt} \% \mathrm{Si}-\mathrm{OH}$ bonds.

A similar effect can be observed in the case of Fe incorporation. The incorporation of only $5 \mathrm{Mol} \% \mathrm{Fe}$ reduces the activation energy $E_{\mathrm{a}} / k$ by about $2000-3000 \mathrm{~K}$ in comparison with the iron-free magnesium silicate. This is due to the decrease of viscosity beyond the transformation range of the amorphous material. The calculation of the activation energy enables us to compare the crystallization behaviour of different materials and to estimate time scales necessary for crystallization at different temperatures. For example, a magnesium silicate without Si-OH groups needs $\tau=3300$ yrs for crystallization at a temperature of $750 \mathrm{~K}$, whereas $\mathrm{Si}-\mathrm{OH}$-containing magnesium silicates crystallize in minutes up to hours at this temperature. This time scale matches well to the expected residence times of condensed silicates close to the condensation zone. The formation of $\mathrm{Si}-\mathrm{OH}$ bonds in cosmic magnesium silicate particles has to be taken into account since $\mathrm{H}_{2} \mathrm{O}$ as the most abundant oxygenbearing molecule plays a very important role in the condensation of circumstellar silicates (Gail \& Sedlmayr 1998).

\section{Comparison with observations}

The prediction of the formation of pure magnesium or iron silicates during gas phase condensation in the $\mathrm{MgO} \cdot \mathrm{FeO} \cdot \mathrm{SiO}_{2}$ system and the formation of eutectic compositions (Rietmeijer et al. 1999a,b) raised a lot of questions concerning the production of amorphous mixed $\mathrm{Mg}-\mathrm{Fe}$ silicates. The condensation of an amorphous pure magnesium silicate has been made likely by the detection of only very pure crystalline magnesium silicates which can be formed from the corresponding amorphous precursor by thermal annealing (Hallenbeck et al. 1998; Fabian et al. 2000). This scenario is also supported by gas phase condensation experiments which have shown that silicates are originally formed in the amorphous state. Though the activation energy of crystallization is smaller for iron-containing silicates due to the decrease of viscosity compared to magnesium silicates, there is no observational evidence for the formation of crystalline Fe-silicates in space. There have to be good reasons for the formation of only crystalline magnesium silicates.

\subsection{AGB stars}

The testbed of silicate dust laboratory analogues is the comparison of theoretical spectra based on such data with the observed spectral energy distributions (SED). The broad and mostly structureless appearance of the emission profiles of the circumstellar silicate bands of evolved stars at 10 and $18 \mu \mathrm{m}$ inhibits direct mineralogical conclusions. There are, however, characteristic points that have some diagnostic value: the exact peak positions and the widths of the bands, the asymmetry of the $10 \mu \mathrm{m}$ band profile, the ratio of the band strengths, and the depth and shape of the "trough" between them. The exact appearance of the absorption coefficient derived from the observed SED is sensitively influenced by the assumptions of the derivation procedure (photospheric model of the star, assumed dust temperature, points where the dust emissivity of the envelope vanishes). An important point is that there could be a continuous dust absorption superposed to the silicate absorption bands. This effect could mask the widths of the bands and the absorption between the bands, in the so-called trough.

For the comparison with the observations we chose the two AGB stars TY Draconis and R Cassiopeiae, for which high-quality ISO-SWS spectra in the wavelength range from 2 to $45 \mu \mathrm{m}$ are available. These spectra have been downloaded from the ISO archive and were reduced with the ISO Spectral Analysis Package (ISAP). TY Dra shows very slender bands and a deep trough, whereas the Mira star R Cas represents the case of very broad bands and a shallow trough. As effective dust temperature we adopted $400 \mathrm{~K}$, that corresponds to the values derived in the literature for Miras and semi-regular variables (Hron et al. 1997).

The spectra of R Cas and TY Dra have been used for this purpose, not only because of the characteristic difference in the MIR spectra of both stars, but also because of the fact that for both of them, several ISO spectra exist, dating from different phases in their cycles of variability. The different spectra of $\mathrm{R}$ Cas strongly resemble each other in the region dominated by the dust emission (Kerschbaum et al. 2000), and the same is true for the spectra of TY Dra. This indicates that intrinsic differences between both stars are responsible for their largely different MIR dust emission. 


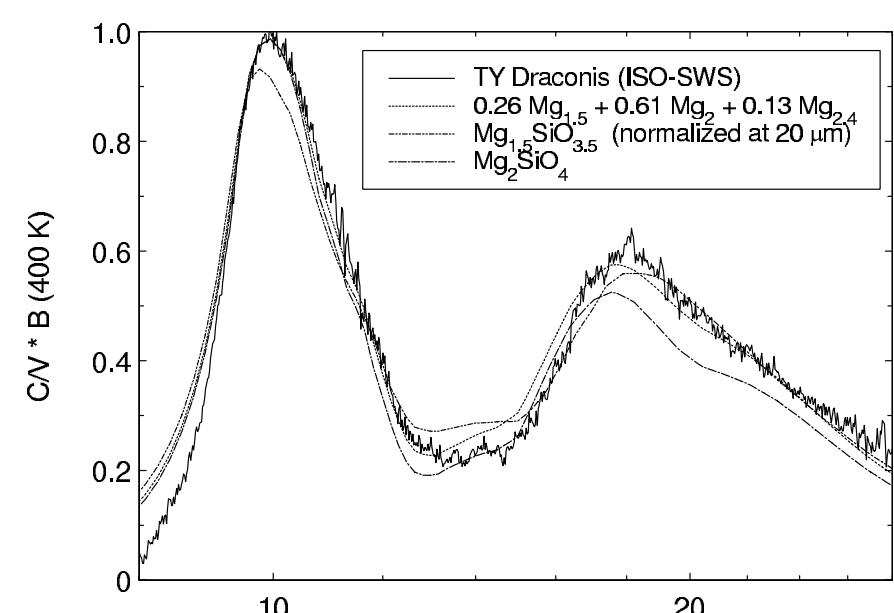

$\lambda(\mu \mathrm{m})$

Fig. 8. Comparison of our sol-gel-produced magnesium silicates with the observed ISO-SWS spectrum of TY Draconis. In the second line of the insert the full silicate formulae have been truncated in order to save space. The mixture of $\mathrm{Mg}_{1.5} \mathrm{SiO}_{3.5}, \mathrm{Mg}_{2} \mathrm{SiO}_{4}$, and $\mathrm{Mg}_{2.4} \mathrm{SiO}_{4.4}$ (for a $\mathrm{CDE}$ ) gives the best reproduction of the dust emissivity reached so far.

The dust emissivity has been derived in the following way: At $\lambda=7 \mu \mathrm{m}$, the observed flux was assumed to be wholly dominated by the stellar photosphere. Therefore, a Rayleigh function has been fitted to the spectral energy distribution at this wavelength and has been subtracted from the SED in order to derive the dust emissivity. Subtracting more sophisticated photospheric models which take into account the molecular absorption (primarily due to $\mathrm{H}_{2} \mathrm{O}, \mathrm{CO}$ and $\mathrm{SiO}$ ) does not lead to significantly different results for the dust emissivity in this case, especially not for the region $\lambda>9 \mu \mathrm{m}$ in which we are primarily interested. It is clear from radiative transfer calculations that for stars like TY Dra, the contribution of the dust emission to the total SED amounts to already $5-10 \%$ in the $7 \mu \mathrm{m}$ region. Therefore, the subtraction procedure explained above introduces a small uncertainty of the dust emissivity in the 7-9 $\mu \mathrm{m}$ range. In Fig. 8, the normalized dust spectrum of TY Dra obtained by the described procedure is compared with emissivities determined from the calculated absorption coefficients of the sol-gel silicates. While the observed spectrum cannot be satisfactorily reproduced by using a single magnesium silicate compound, a mixture of $\mathrm{Mg}_{1.5} \mathrm{SiO}_{3.5}, \mathrm{Mg}_{2} \mathrm{SiO}_{4}$ and $\mathrm{Mg}_{2.4} \mathrm{SiO}_{4.4}$ gives an almost perfect agreement with the observation. This shows that pure amorphous magnesium silicates are the best candidates for the dust spectra of such stars. The biggest deviation is visible at the "blue" wing of the $10 \mu \mathrm{m}$ band. The corresponding dust emissivity of R Cas (see Fig. 11) cannot be exclusively represented by sol-gel magnesium silicates or by the glassy $\mathrm{Mg}-\mathrm{Fe}$-silicates. This is mainly due to $\mathrm{R}$ Cas' enhanced emission in the $11-15 \mu \mathrm{m}$ range, which is discussed below.

One problem with the magnesium silicates is their very low NIR opacity, which inhibits effective absorption of the stellar radiation and therefore strongly reduces heating. However, taking into account the probable formation of metallic iron in

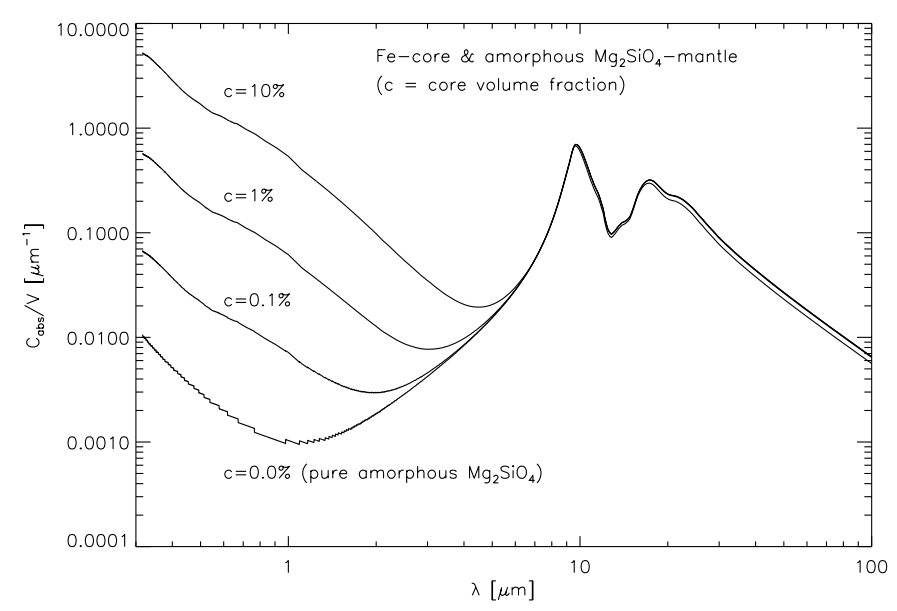

Fig. 9. The effect of metallic iron impurities on the absorption efficiency $Q_{\text {abs }} / a$ of amorphous $\mathrm{MgSiO}_{4}$. The curves shown here have been calculated by considering the Fe inclusions as cores of coremantle-particles.

oxygen-rich circumstellar shells (see e.g. Kozasa \& Hasegawa 1988; Gail \& Sedlmayr 1999), this problem can be easily solved, because metallic iron inclusions will strongly enhance the NIR absorption of Mg silicates as recently demonstrated by Kemper et al. (2002). Near $1 \mu \mathrm{m}$, where the opacity of pure magnesium silicates reaches its minimum, the value of $Q_{\text {abs }} / \mathrm{a}$ is increased by a factor of about 10 if only $0.1 \%$ of the grain volume is a metallic Fe inclusion. An enhancement by a factor of 100 is reached if $1 \%$ of the grain volume is metallic Fe (see Fig. 9).

It is a well-known fact that the silicate spectra of AGB-stars and other evolved stars exhibit great spectral diversity especially in the $10 \mu \mathrm{m}$ region. Speck et al. (2000) have used UKIRT CGS3 spectra in order to classify the $10 \mu \mathrm{m}$ profiles of AGB stars and supergiants into characteristic groups. We examined whether our magnesium silicates are able to match the mean spectra of selected star groups. Figure 10 illustrates that the derived mean profiles of group $\mathrm{A}$ to $\mathrm{C}$ and the additional supergiant group 1 can be well reproduced either by mixtures of or single magnesium silicates. The mean spectrum of supergiant silicate group 1 can be matched by $\mathrm{Mg}_{1.5} \mathrm{SiO}_{3.5}$ spheres in the Rayleigh limit. The spectrum of group A of the AGB stars can be fitted by either a single magnesium silicate $\left(\mathrm{Mg}_{1.5} \mathrm{SiO}_{3.5}\right)$ or a mixture of the two components $\mathrm{Mg}_{2} \mathrm{SiO}_{4}$ and $\mathrm{Mg}_{2.4} \mathrm{SiO}_{4.4}$. In contrast groups $\mathrm{C}$ and $\mathrm{D}$ require a rather complex mixture with varying $\mathrm{Mg} / \mathrm{Si}$ ratios.

\subsection{The trough opacity problem}

When comparing the dust emission of the circumstellar envelope of TY Dra with that of a typical Mira variable like R Cas or R Aql, it becomes clear that the flux minimum between the two silicate bands is much less conspicuous than should be expected from the absorption efficiency of almost any amorphous silicate produced in the laboratory. This discrepancy is known for several years already; it has been called the "trough opacity problem" (Dorschner 1999). 


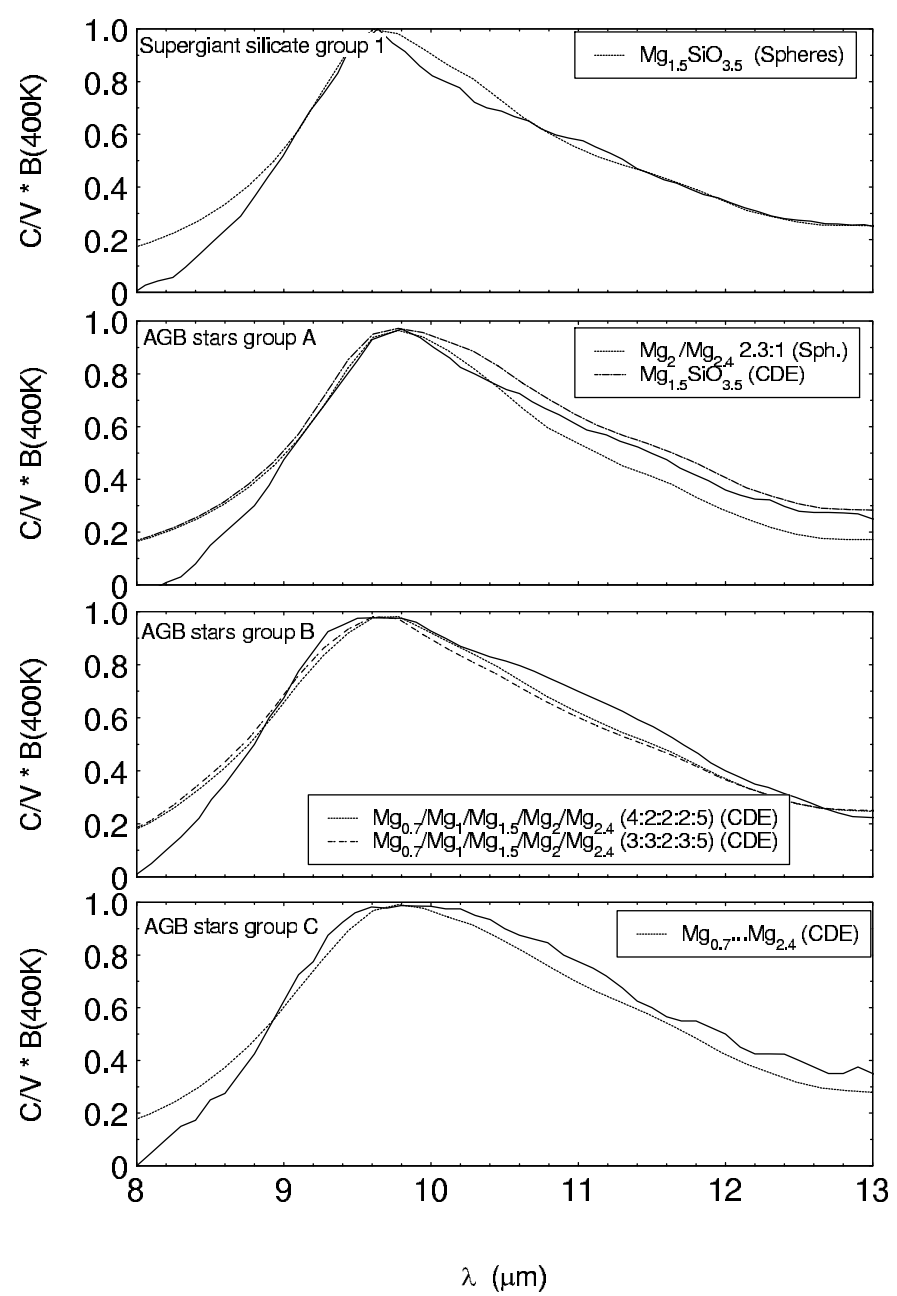

Fig. 10. Reproduction of the mean spectra of AGB star groups A, B, C and supergiants group 1 (Speck et al. 2000) with sol-gel magnesium silicates of different stoichiometry. The solid lines represent the star spectra and the dashed or dotted lines stand for either single or mixtures of magnesium silicates. The AGB stars group C profile (lowest panel) equal portions of all magnesium silicates have been mixed.

It turned out that "filling of the trough" in order to obtain spectra like that of $\mathrm{R}$ Cas cannot be reached by silicate absorption. Silicates containing alkaline earth or transition metal oxides cannot provide spectra with $10 \mu \mathrm{m}$ profiles shifted to wavelengths longer than $11 \mu \mathrm{m}$ since the position of this $\mathrm{Si}-\mathrm{O}$ stretching band depends on the polymerization degree of $\mathrm{SiO}_{4}$ tetrahedra and therefore on the number of bridging oxygen atoms in the silicate network (McMillan 1984). Even though in $\mathrm{Mg}_{2} \mathrm{SiO}_{4}$ only non-bridging oxygen atoms are present, the formation of a three-dimensional amorphous network requires the existence of at least a small portion of bridging oxygen atoms. This can only be compensated by the incorporation of structural inhomogeneity on an atomic scale. A considerably higher content of metal oxide in the silicate gives rise to the formation of rather inhomogeneous material due to the stoichiometry problems. In this study we were able to incorporate a metal oxide content up to $2.4 \mathrm{wt} \%$ without producing a non-homogeneous material.

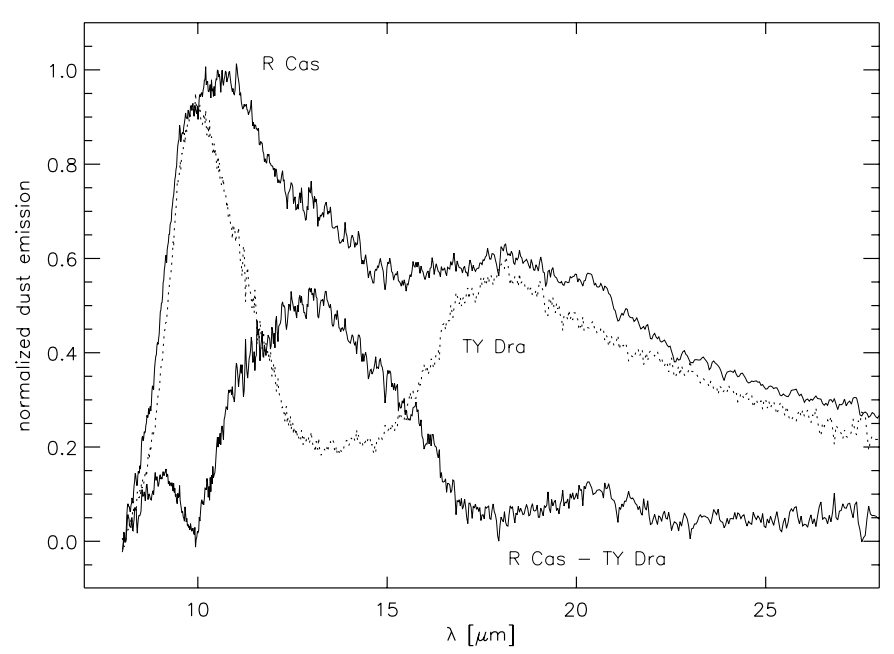

Fig. 11. Difference between the normalized dust emissivities of R Cas and TY Dra. The broad maximum around $13 \mu \mathrm{m}$ is supposed to be caused by hitherto unidentified dust components which do not belong to the group of pure magnesium or magnesium-iron silicates.

There are, however, oxides that could contribute to the trough opacity. First observational evidence pointing in this direction was given by the $13 \mu \mathrm{m}$ band that has been discussed as being due to $\mathrm{Al}-\mathrm{O}$ vibrations in $\alpha-\mathrm{Al}_{2} \mathrm{O}_{3}$ or in $\mathrm{MgAl}_{2} \mathrm{O}_{4}$ (Begemann et al. 1997; Posch et al. 1999; Fabian et al. 2001). Further experiments with other oxides that could effectively contribute to the trough opacity are in progress in the Jena laboratory.

In order to roughly illustrate the amount and the cumulative SED of potential trough opacity contributors from the observations, we simply subtracted the normalized dust emission of a star like TY Dra from the normalized dust emission of a star that does not show a deep trough between the two silicate bands such as R Cas. The result of such a subtraction is shown in Fig. 11. As already mentioned, this difference spectrum can hardly be understood in terms of different silicate dust populations. Different grain sizes could play a role, since for large grains (i.e. when their radius is no longer small compared to the wavelength), both the 10 and the $20 \mu \mathrm{m}$ bands can develop huge shoulders partly filling the trough between them. However, the dominance of grains with diameters of several $\mu \mathrm{m}$ is not very probable in an average circumstellar shell. An exception are very evolved AGB or massive stars, which can have grain sizes up to $6 \mu \mathrm{m}$ (Molster et al. 1999; Hoogzaad et al. 2002). Optical depth effects are negligible, because the circumstellar shells of both stars are optically thin over the whole wavelength range considered here. Therefore, it seems rather likely that a distinct dust grain population is responsible for the broad emission peaking around $13 \mu \mathrm{m}$. As potential carriers of this emission oxides like $\mathrm{Al}_{2} \mathrm{O}_{3}$ (Begemann et al. 1997), $\mathrm{MgAl}_{2} \mathrm{O}_{4}$ (Posch et al. 1999; Fabian et al. 2001) and $\mathrm{Ca}-\mathrm{Al}$-oxides (Mutschke et al. 2002) have been discussed. It is an interesting coincidence that the only band that has been definitely observed is located exactly at the $13 \mu \mathrm{m}$ peak of the difference spectrum. 


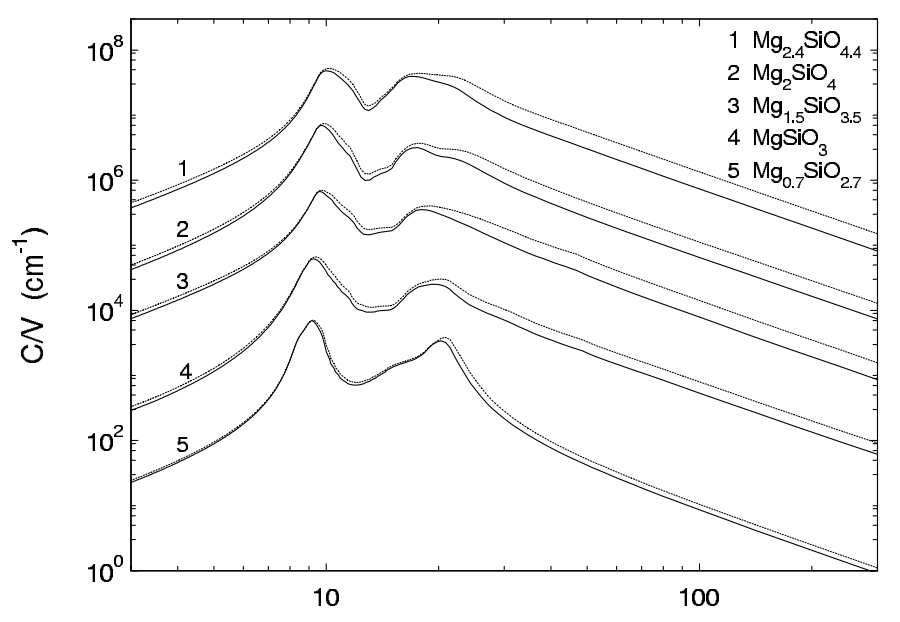

$\lambda(\mu \mathrm{m})$

Fig. 12. Calculated absorption cross sections per unit volume of the magnesium silicates for spheres (solid lines) and CDE (dashed lines).

\subsection{The FIR opacity}

The spectral behaviour of cosmic dust in the FIR deserves special interest with respect to astrophysical questions. Submillimeter observations provide a direct possibility for estimating mass loss rates and the total mass of circumstellar or interstellar dust. The calculated absorption efficiency for spheres and a CDE in the FIR range follows a power law with the exponent -2 . Small differences in the long-wavelength extrapolation do not influence the spectral index. The spectral index of pyroxene and olivine glasses has a very similar value.

\section{Conclusions}

In contrast to composite magnesium-, iron-, calcium- and sodium-silicates, pure amorphous $\mathrm{Mg}$-silicates with various $\mathrm{Mg} / \mathrm{Si}$ ratios have not yet been investigated systematically. However, these substances are of importance in the context of interstellar silicate mineralogy, since they are potential precursors of the crystalline $\mathrm{Mg}$-silicates discovered by ISO in the spectra of circumstellar shells.

By means of the sol-gel technique, we were able to produce amorphous magnesium silicates with different $\mathrm{Mg} / \mathrm{Si}$ ratios in a homogeneous form and to derive optical constants by Kramers-Kronig analysis and Lorentz-oscillator fit method. The diagnostic spectral parameters in the MIR region have been derived by calculating the absorption efficiency in vacuum both for spheres and for a distribution of ellipsoids in the Rayleigh limit. The 10 and the $20 \mu \mathrm{m}$ bands have been shown to approach each other with increasing magnesium content (which shifts the $10 \mu \mathrm{m}$ band towards the "red" and the $20 \mu \mathrm{m}$ band towards the "blue"). This effect is due to the influence of the $\mathrm{MgO}$ content on the degree of polymerization of the $\mathrm{SiO}_{4}$ tetrahedra. As expected, large differences between pure magnesium- and composite magnesium-ironsilicates were found in the NIR region.

The crystallization of amorphous Mg-silicates by thermal annealing has also been investigated. The sol-gel produced magnesium silicates show an interesting annealing behaviour. The activation energy was found to be independent of the $\mathrm{Mg} / \mathrm{Si}$ ratio, but largely dependent on the $\mathrm{Si}-\mathrm{OH}$ content. The activation energy of crystallization can be reduced by one third by the presence of up to $1.5 \mathrm{wt} \% \mathrm{Si}-\mathrm{OH}$ bonds compared to magnesium silicates which do not contain $\mathrm{Si}-\mathrm{OH}$. This leads to a significant reduction of the necessary crystallization temperature and crystallization time.

First results of a comparison with observed dust emission spectra underline the relevance of amorphous $\mathrm{Mg}$-silicates for our understanding of circumstellar shells. Comparisons with the normalized dust spectrum of TY Dra have demonstrated that a mixture of $\mathrm{Mg}_{1.5} \mathrm{SiO}_{3.5}, \mathrm{Mg}_{2} \mathrm{SiO}_{4}$ and $\mathrm{Mg}_{2.4} \mathrm{SiO}_{4.4}$ gives a nearly perfect match with the observations. Pure amorphous magnesium silicates are the best candidates for modeling the dust spectra of such stars.

The corresponding dust emissivity of R Cas (see Fig. 11) cannot be exclusively represented by sol-gel magnesium silicates or by the glassy $\mathrm{Mg}-\mathrm{Fe}$-silicates. Other dust components like aluminium oxides or spinels can contribute to the trough opacity. The trough opacity beyond $16 \mu \mathrm{m}$ could be influenced by iron oxide or mixed magnesium iron oxides. Furthermore, different grain sizes could play a role, since for large grains (i.e. when their radius is no longer small compared to the wavelength), both the 10 and the $20 \mu \mathrm{m}$ bands can develop huge shoulders partly filling the trough between them. However, the dominance of grains with diameters of several $\mu \mathrm{m}$ is not very probable in an average circumstellar shell.

Acknowledgements. This work has been supported by the Deutsche Forschungsgemeinschaft (DFG grant DO 575/2-3). TP received a DOC grant by the Austrian Academy of Sciences. We gratefully thank A.K. Speck for providing us $10 \mu \mathrm{m}$ spectra of oxygen-rich evolved stars for comparison. Furthermore, we would like to thank G. Born for her help in the laboratory.

\section{References}

Aasland, S., Einarsrud, M.-A., Grande, T., Grzechnik, A., \& McMillan, P. F. 1997, J. Non-Cryst. Sol., 213, 341

Agladze, N. I., Sievers, A. J., Jones, S. A., Burlitch, J. M., \& Beckwith, S. V. W. 1996, ApJ, 462, 1026

Begemann, B., Dorschner, J., Henning, T., et al. 1997, ApJ, 476, 199

Bohren, C. F., \& Huffman, D. R. 1983, Absorption and Scattering of Light by Small Particles (John Wiley and Sons, Inc.)

Bouwman, J., Meeus, G., de Koter, A., et al. 2001, A\&A, 375, 950

Bowey, J. E., Barlow, M. J., Molster, F. J., et al. 2002, MNRAS, 331, L1

Bowey, J. E., Lee, C., Tucker, C., et al. 2001, MNRAS, 325, 886

Brinker, C. J., \& Scherer, G. W. 1990, Sol-Gel Science (Boston: Academic Press)

Burlitch, J., Beeman, M., Riley, B., \& Kohlstedt, D. 1991, Chem. Mater., 3, 692

Crovisier, J., Leech, K., Bockelee-Morvan, D., et al. 1997, Science, 275, 1904

Demyk, K., Jones, A. P., Dartois, E., Cox, P., \& d'Hendecourt, L. 1999, A\&A, 349, 267

Dorschner, J. 1999, in Formation and evolution of solids in space, ed. J. M. Greenberg, \& A. Li (Dordrecht: Kluwer), 229

Dorschner, J., Begemann, B., Henning, T., Jäger, C., \& Mutschke, H. 1995, A\&A, 300, 503 
Dorschner, J., \& Henning, T. 1986, Ap\&SS, 128, 47

Dorschner, J., \& Henning, T. 1995, A\&ARv, 6, 271

Draine, B. T., \& Lee, H. M. 1984, ApJ, 285, 89

Efimov, A. M. 1999, J. Non-Cryst. Sol., 253, 95

Eriksson, T. S., Hjortsberg, A., Niklasson, G. A., \& Granqvist, C. D. 1981, Appl. Opt., 20, 2742

Fabian, D., Jäger, C., Mutschke, H., Dorschner, J., \& Henning, T. 2000, A\&A, 364, 282

Fabian, D., Posch, T., Mutschke, H., Kerschbaum, F., \& Dorschner, J. 2001, A\&A, 373, 1125

Fuxi, G. 1979, Optical and spectroscopic properties of glass (Berlin: Springer Verlag)

Gail, H. P., \& Sedlmayr, E. 1998, in The molecular astrophysics of stars and galaxies, ed. T. W. Hartquist, \& D. A. Williams (Oxford: Clarendon Press), 285

Gail, H. P., \& Sedlmayr, E. 1999, A\&A, 347, 594

Gaskell, P. H., \& Johnson, D. W. 1976, J. Non-Cryst. Sol., 20, 153

Gervais, F., Blin, A., \& Massiot, D., et al. 1987, J. Non-Cryst. Sol., 89,384

Hallenbeck, S. L., Nuth, J. A., \& Daukantas, P. L. 1998, Icarus, 131, 198

Hallenbeck, S. L., Nuth, J. A., \& Nelson, R. N. 2000, ApJ, 535, 247

Hoogzaad, S. N., Molster, F. J., Dominik, C., et al. 2002, A\&A, 389, 547

Hron, J., Aringer, B., \& Kerschbaum, F. 1997, A\&A, 322, 280

Hudgens, J. J., \& Martin, S. W. 1996, Phys. Rev. B, 53, 5348

Iler, R. K. 1979a, The chemistry of silica (New York: John Wiley \& Sons), 622

Iler, R. K. 1979b, The chemistry of silica (New York: John Wiley \& Sons), 546

Jäger, C., Molster, F. J., Dorschner, J., et al. 1998, A\&A, 339, 904

Jäger, C., Mutschke, H., Begemann, B., Dorschner, J., \& Henning, T. 1994, A\&A, 292, 641

Justtanont, K., De Jong, T., Helmich, F. P., et al. 1996, A\&A, 315, 217

Kamitsos, E. I., Kapoutsis, G. A., Chryssikos, G. D., et al. 1995, Phys. Chem. Glasses, 36, 141

Kemper, F., de Koter, A., Waters, L. B. F. M., Bouwman, J., \& Tielens, A. G. G. M. 2002, A\&A, 384, 585

Kerschbaum, F., Posch, T., \& Aringer, B. 2000, in ISO beyond the peaks: The 2nd ISO workshop on analytical spectroscopy, ed. A. Salama, M. F. Kessler, K. Leech, \& B. Schulz, ESA-SP, 456, 203
Kozasa, T., \& Hasegawa, H. 1988, Icarus, 73, 180

Lenzuni, P., Gail, H. P., \& Henning, T. 1995, ApJ, 447, 848

Liepmann, M. J., \& Neuroth, N. 1995, in The properties of optical glass, ed. H. Bach, \& N. Neuroth (Berlin: Springer Verlag), 290

McMillan, P. 1984, Amer. Mineral., 69, 622

Messenger, S., Keller, L. P., \& Walker, R. M. 2002, 33rd Annual Lunar and Planetary Science Conf., March 11-15, 2002, Houston, Texas, abstract No. 1887, 33

Mitra, S. S. 1962, Sol. State Phys., 13, 1

Molster, F. J., Waters, L. B. F. M., \& Tielens, A. G. G. M. 2002a, A\&A, 382, 222

Molster, F. J., Waters, L. B. F. M., Tielens, A. G. G. M., Koike, C., \& Chihara, H. 2002b, A\&A, 382, 241

Molster, F. J., Waters, L. B. F. M., Trams, N., et al. 1999, A\&A, 350, 163

Morimoto, Y., Nozawa, S., \& Hosono, H. 1999, Phys. Rev. B, 59, 4066

Mutschke, H., Begemann, B., Dorschner, J., et al. 1997, A\&A, 333, 188

Mutschke, H., Posch, T., Fabian, D., \& Dorschner, J. 2002, A\&A, 392, 1047

Nuth, J. A. 1996, in The Cosmic Dust Connection, ed. J. M. Greenberg, NATO ASI Ser. (Dordrecht: Kluwer), 205

Ossenkopf, V., Henning, T., \& Mathis, J. S. 1992, A\&A, 261, 567

Posch, T., Kerschbaum, F., Mutschke, H., et al. 1999, A\&A, 352, 609

Rietmeijer, F. J. M., Nuth, J. A., \& Karner, J. M. 1999a, ApJ, 527, 395

Rietmeijer, F. J. M., Nuth, J. A., \& Karner, J. M. 1999b, Phys. Chem. Chem. Phys., 1, 1511

Scholze, H. 1988, Glas (Berlin: Springer Verlag), 136

Scott, A., \& Duley, W. W. 1996, Ap\&SS, 105, 401

Shinoda, K., Yamakata, M., Nanba, T., et al. 2002, Phys. Chem. Miner., 29, 396

Speck, A. K., Barlow, M. J., Sylvester, R. J., \& Hofmeister, A. M. 2000, A\&AS, 146, 437

Waelkens, C., Waters, L. B. F. M., de Graauw, M. S., et al. 1996, A\&A, 315, L245

Waters, L. B. F. M., Molster, F. J., de Jong, T., et al. 1996, A\&A, 315, L361

Waters, L. B. F. M., Morris, P. W., Voors, R. H. M., \& Lamers, H. J. G. L. M. 1997, ed. A. Nota, \& H. J. G. L. M. Lamers (San Francisco: Astron. Soc. Pacific), ASP Conf. Ser., 120, 326

Wooten, F. 1972, Optical properties of solids (New York: Academic Press), 42 